\title{
Patterns of central nervous system complications of post-hematopoietic stem cell transplant in pediatric oncology patients: a single institute experience
}

Samar Ahmed Hussein ${ }^{1 *}$ (D) Mahmoud Hammad ${ }^{2}$, Amr Abdalla $^{2}$, Khaled Alsheshtawi ${ }^{3}$, Iman Mohamed Zaky ${ }^{1}$ and Ayda Youssef ${ }^{1}$

\begin{abstract}
Background: Hematopoietic stem cell transplant (HSCT) has been increasingly used in the last few decades, with improved success in offering a cure. CNS complications are an important contributor to morbidity and mortality in HSCT patients. The aim of the study was to evaluate the role of imaging in the detection and assessment of CNSC (central nervous system complications) after HSCT in pediatric oncology patients.

The study included consecutive pediatric patients who underwent HSCT for hematologic or solid malignancies at CCHE-57357 (Children Cancer Hospital-Egypt 57357) from January 2011 to March 2019. The age of the patients in the study ranged from 0.9 to 25 years (median age 6.5 years). CT (computed tomography) and/or MRI (magnetic resonance imaging) studies were evaluated for the detection and characterization of CNSC.

Results: The incidence of post-HSCT CNSC was 13\% with a day 100 and 5-year cumulative incidence of 9.3 and $12.5 \%$, respectively. The most commonly observed CNSC detected was disease recurrence, followed by PRES (Posterior reversible encephalopathy syndrome). CNS recurrence of the initial diagnosis, atrophy, and infection were more common at the $>100$-day post-HSCT transplant period, while PRES was much more common at $<100$-day post-HSCT.

Conclusion: CNS complications are an important contributor to morbidity and mortality in HSCT patients that require MRI protocols distinctively tailored for each patient, clinical suspicion, and proper imaging assessment for early detection and follow-up.
\end{abstract}

Keywords: Hematopoietic stem cell transplant, Allogenic, Autologous, CNS, Complications, PRES

\section{Background}

Hematopoietic stem cell transplantation (HSCT) is a highly effective treatment for various benign and malignant diseases [1]. Few studies are available in the pediatric age group describing the incidence of posttransplant central nervous system complications (CNSC)

\footnotetext{
* Correspondence: samar.ahmad@nci.cu.edu.eg

'Department of Diagnostic and Interventional Radiology, National Cancer Institute, Faculty of Medicine, Cairo University and Children's Cancer Hospital (CCHE-57357), Cairo, Egypt

Full list of author information is available at the end of the article
}

and ranged from 8 to $65 \%$, being the principal causes of death in 9 to $17 \%$ of the cases $[2,3]$.

The incidence of neurological CNS complications was highest in unrelated allogeneic transplantations (39\%), followed by matched related allogeneic (21\%) and autologous transplantations (11\%) [4].

Post-transplant CNS complications are categorized into five major groups: cerebrovascular, metabolic, infectious, therapy-induced disorders, and post-HSCT carcinogenesis [3].

\section{Springer Open}

(-) The Author(s). 2021 Open Access This article is licensed under a Creative Commons Attribution 4.0 International License, which permits use, sharing, adaptation, distribution and reproduction in any medium or format, as long as you give appropriate credit to the original author(s) and the source, provide a link to the Creative Commons licence, and indicate if changes were made. The images or other third party material in this article are included in the article's Creative Commons licence, unless indicated otherwise in a credit line to the material. If material is not included in the article's Creative Commons licence and your intended use is not permitted by statutory regulation or exceeds the permitted use, you will need to obtain permission directly from the copyright holder. To view a copy of this licence, visit http://creativecommons.org/licenses/by/4.0/. 
The frequency and diversity of neurologic complications largely depend on multiple risk factors, including pre-transplant disease risk status, types of the conditioning regimen, post-transplant immunosuppressive regimens, pancytopenia, coagulopathy, and greater than grade II graft versus host disease (GVHD) [5, 6].

With the global increase in using HSCT, it requires a high level of diligence for radiologists to be familiar with CNS complications, their relationship to the patient's immune status, and their imaging appearances $[3,7]$.

\section{Methods}

\section{Patients}

The study included consecutive pediatric patients who underwent HSCT at CCHE-57357 from January 2011 to March 2019. The study retrospectively followed up 525 HSCT patients. Median follow-up for survivors was 48 months (range 6-109 months). The institutional review board approved this study.

\section{Imaging}

MRI brain studies were obtained at $1.5 / 3 \mathrm{~T}$ with a standard head-coil.

All patients were submitted to the standard MRI protocol utilizing 1.5/3 $\mathrm{T}$ scanner including the following MR sequences: multi-planar MR imaging sequences without contrast including T1 and T2 WI, gadoliniumenhanced MR imaging with dynamic sequence acquisition (whenever indicated), diffusion-weighted sequence with multiple b-values with ADC (apparent diffusion coefficient) mapping (whenever indicated), magnetic resonance venography (MRV) (whenever indicated).

CT imaging was performed as initial or complementary examination whenever possible or required, with or without intravenous contrast administration.

\section{Imaging evaluation and data analyses}

Brain imaging structural abnormalities were defined in the following categories: cerebrovascular complications (including intra-parenchymal hemorrhage, venous thrombosis), CNS infection, CNS recurrence of hematological malignancy, atrophy, metabolic abnormalities, and drug toxicity such as PRES and leukoencephalopathy. CNSC that occurred after relapse or progression of underlying diseases were excluded from the analysis.

Radiological findings were reviewed by 2 radiologists who retrospectively independently reviewed patients' imaging. Transplant data were collected from the CCHE transplant unit and included the type of transplant, conditioning, and immunosuppressive regimen; neurologic symptoms and its onset; and imaging methods. Diagnosis of CNSC was made using clinical, radiological, pathological, and/or microbiologic data (if available). The onset of complications reported as < 30 days, 30-100 days, or $>100$ days post-transplant.

\section{Statistical analyses}

Descriptive statistics were performed for all variables. Continuous data reported as means with standard deviations. All categorical data were reported as percentages. Student $t$ test or Mann-Whitney $U$ test (for nonparametric distributions) was used to compare continuous variables. Chi-square test was used for the comparison of categorical variables. The significance level was set at a $P$ value of _0.05 (two-sided). We performed a logistic regression analysis to estimate the odds ratio and $95 \%$ confidence intervals (CIs). Stepwise multivariate logistic regression was used to understand the independent role of variables in predicting CNSC for patients who underwent allogeneic and autologous HSCT. Statistical analysis was performed using statistical software: SPSS statistical package version 20 .

\section{Results}

Patients characterization

A total of 525 cases were included of whom 311 and 214 had undergone AuSCT (Autologous stem cell transplant) and AlloSCT (Allogenic stem cell transplant), respectively. A detailed categorization of patients are mentioned in Fig. 1. Patient and transplant details are shown in Table 1.

\section{Incidence}

The incidence of post-HSCT CNSC is $13 \%$. Cumulative frequency of CNSC was revealed at 100 days and 5-year incidence of $5.3 \%$ and $12.5 \%$, respectively (Table 2 ). Sixty-eight (68) out of a total of 525 patients (13\%) presented with a sum of 77 episodes of CNSC.

\section{Type and time of onset of CNS complications}

The most commonly observed CNSC was CNS disease recurrence in 28/77 (36.4\%), followed by PRES detected in 17/77 (22\%) episodes. The incidence of other CNSC are mentioned in Table 3.

CNS recurrence of the initial diagnosis, atrophy, and infection were more common at the $>100$ days postHSCT transplant period, while PRES was much more common at $<100$ days post-HSCT.

\section{Risk factors}

Prior post-therapeutic CNSC (including leukoencephalopathy, PRES, CNS hemorrhage, atrophy, and CNS infection) statistically proved to be an independent risk factor for post-AuSCT CNSC with 6.7 times increased risk ( $P$ value $<0.001$ ).

Females proved to be 2.3 times more susceptible to post-AlloSCT CNSC ( $P$ value 0.02 ); however, sex failed 


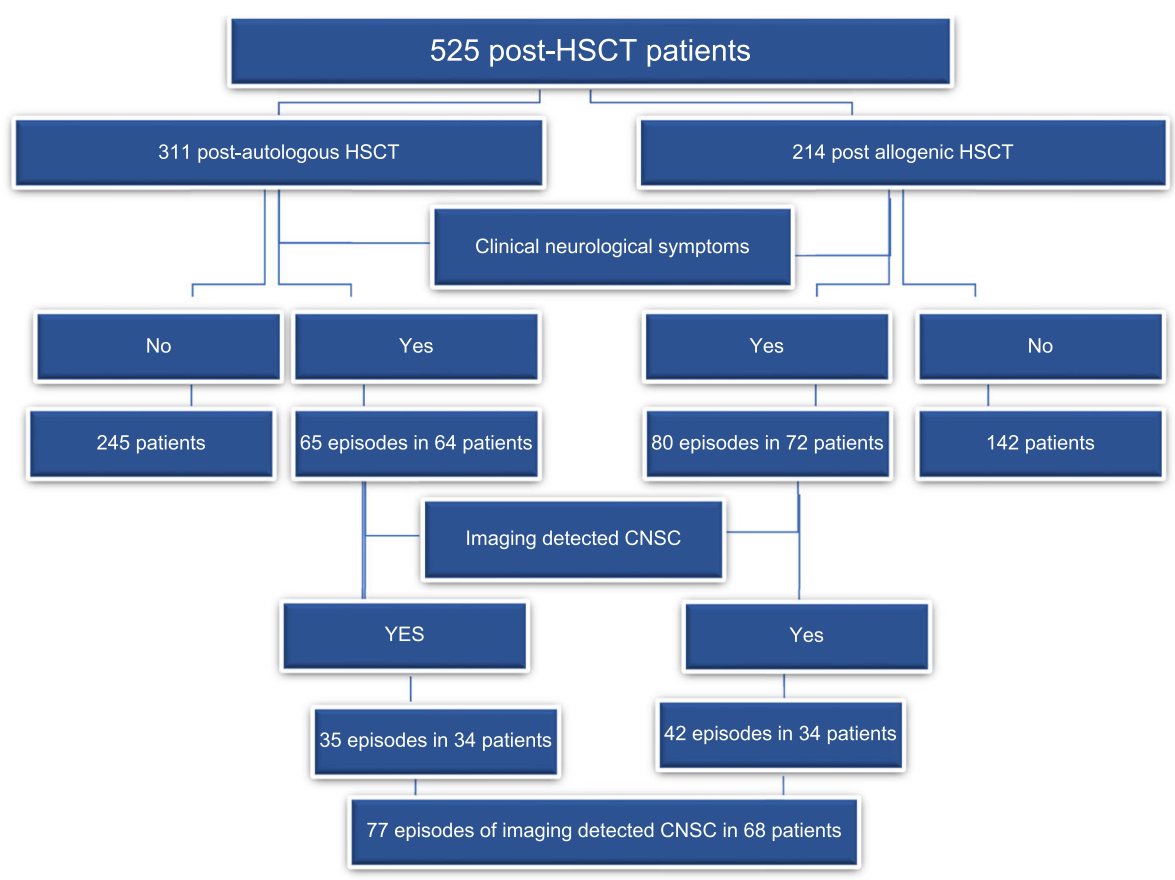

Fig. 1 Categorization of the HSCT patients included in the study

to be an independent risk factor of post-AlloSCT CNSC. Post-AlloSCT patients with acute GVHD are 4.3 times susceptible to developing post-transplant CNSC in both univariate and multivariate analysis ( $P$ value 0.002$)$.

Otherwise, no differences in patient demographics, disease type, disease status pre-transplant, and transplant-related characteristics were noted between those with and without CNS complications.

\section{Clinical presentation}

A total number of 145 clinical neurological symptom episodes were detected. Regarding clinical neurological symptoms with corresponding imagingdetected CNS complications, headache had the highest sensitivity (48.7\%), while the highest specificity belongs to visual symptoms and impaired conscious level showing the specificity of 88.7 and $87.3 \%$, respectively.

\section{Allogenic versus autologous groups \\ Higher incidence of post-HSCT CNS complications were recorded in the allogenic HSCT group (15.9\%) compared with autologous the HSCT group (11\%).}

\section{Imaging}

CNS recurrence (Figs. 2 and 3 )

The incidence of post-HSCT CNS recurrence was $5 \%$. Most CNS recurrence cases (89\%) were post-AuSCT. The post-alloSCT CNS recurrence patients (11\%) presented as disseminated leukemic infiltrations in MR imaging.

Intra-parenchymal masses with or without leptomeningeal affection was the most common form of CNS recurrence seen in $25 / 28$ (82\%) of recurrence cases, while leptomeningeal form represented only $39 \%$ of the recurrence cases. Nearly half of the recurrent cases (45.5\%) showed a hyperintense signal in both T2 \& FLAIR (fluid-attenuated inversion recovery) images with heterogeneous post-contrast enhancement seen in $89.5 \%$ of the cases.

\section{PRES (Fig. 4)}

The incidence of PRES in post-HSCT patients was $3.2 \%$. Females were 4.2 times susceptible to developing PRES than males $(P<0.006)$. Nearly one third (35.5\%) of the PRES episodes were detected in AML (Acute myeloid leukemia) patients. A bilateral asymmetrical imaging pattern was noted in 13/17 (76.5\%) of PRES patients. The most frequent PRES sites were occipital and parietal regions in 15 (88.2\%) and 14 $(82.4 \%)$ patients, respectively. Imaging findings of PRES showed an isointense T1WI (T1-weighted imaging) signal in $64.5 \%$ of cases along with hyperintense T2WI (T2-weighted imaging) \& FLAIR signal in $100 \%$ of cases. Only 5/17 (29.4\%) of PRES cases showed diffusion restriction; however, corresponding ADC images did not show significant hypointense signals as seen in cerebral infarcts (pseudo normalization). 
Table 1 Characteristics of HSCT patients

\begin{tabular}{|c|c|c|c|}
\hline & Total cases & AutoSCT & AlloSCT \\
\hline \multicolumn{4}{|l|}{ Age (in years) } \\
\hline Mean & 8.2 & 7.6 & 9.1 \\
\hline Median & 6.5 & 6 & 8.3 \\
\hline Standard deviation & 5.2 & 5.1 & 5.2 \\
\hline Minimum & 0.9 & 1 & 0.9 \\
\hline Maximum & 25 & 25 & 19.3 \\
\hline \multicolumn{4}{|l|}{ Sex } \\
\hline Female & 190 & 114 & 76 \\
\hline Male & 335 & 197 & 138 \\
\hline Initial diagnosis & No (\%) & No (\%) & No (\%) \\
\hline Neuroblastoma (NB) & $210(40 \%)$ & $208(66.9 \%)$ & $2(0.9 \%)$ \\
\hline$C R$ & & 148 & 1 \\
\hline Others $^{\mathrm{a}}$ & & 60 & 1 \\
\hline Non-Hodgkin lymphoma (NHL) & $21(4 \%)$ & $11(3.5 \%)$ & $10(4.7 \%)$ \\
\hline $1 s t C R$ & & 2 & 1 \\
\hline 2nd $C R$ & & 9 & 9 \\
\hline Acute myeloid leukemia (AML) & $76(14.5 \%)$ & $2(0.6 \%)$ & $74(34.6 \%)$ \\
\hline 1st CR & & 1 & 43 \\
\hline 2nd $C R$ & & 1 & 31 \\
\hline Acute lymphoblastic leukemia (ALL) & $44(8.2 \%)$ & $1(0.32 \%)$ & $43(20.1 \%)$ \\
\hline 1st CR & & 0 & 26 \\
\hline 2nd $C R$ & & 1 & 17 \\
\hline Myelodysplastic/myeloproliferative syndrome & $80(15.2 \%)$ & $0(0 \%)$ & $80(37.3 \%)$ \\
\hline Hemophagocytic lymphohistocytosis (HLH) & $5(1 \%)$ & $0(0 \%)$ & $5(2.3 \%)$ \\
\hline Hodgkin lymphoma (HL) & $77(14.7 \%)$ & $77(24.8 \%)$ & $0(0 \%)$ \\
\hline Refractory & & 63 & \\
\hline Relapsing & & 14 & \\
\hline Relapsing Wilms's & $2(0.4 \%)$ & $2(0.6 \%)$ & $0(0 \%)$ \\
\hline Extraocular retinoblastoma (RB) & $10(1.9 \%)$ & $10(3.2 \%)$ & $0(0 \%)$ \\
\hline Total & $525(100 \%)$ & $311(100 \%)$ & $214(100 \%)$ \\
\hline
\end{tabular}

${ }^{a}$ Others contain very good PR and PR neuroblastoma patients

$C R$ Complete response, $P R$ Partial response

Table 2 Cumulative frequency of CNS complications along the follow-up timeline

\begin{tabular}{lll}
\hline & Number of patients & Cumulative frequency (\%) \\
\hline $\mathbf{3 0}$ days & 14 & 2.6 \\
$\mathbf{1 0 0}$ days & 14 & 5.3 \\
$\mathbf{1}$ year & 21 & 9.3 \\
$\mathbf{5}$ years & 17 & 12.5 \\
$\mathbf{7}$ years & 2 & 12.9 \\
\hline
\end{tabular}

\section{Venous thrombosis}

Total incidence of post-HSCT venous thrombosis is $0.9 \%$. All the venous sinus thrombosis cases involved unilateral affection of the venous sinuses, implicating the transverse and sigmoid sinuses in $40 \%$ of cases each and implicating the internal jugular vein (IJV) in 30\% of cases. Thrombosed venous sinuses showed hyperintense T1WI signal in $60 \%$ of cases and isointense or heterogeneous T2WI and FLAIR signal in $80 \%$ of cases.

\section{Hemorrhage}

The incidence of hemorrhage in post HSCT patients is $1.5 \%(n=8), 75 \%$ were post-AlloSCT. Subdural hemorrhage was detected in $3 / 8$ (37.5\%), while 
Table 3 Incidence of CNS complications > 100 days and > 100 days post-HSCT

\begin{tabular}{lll}
\hline & $\begin{array}{l}\text { Number of CNS events } \mathbf{0 - 1 0 0} \text { days } \\
\text { post-transplant }(\boldsymbol{n}=\mathbf{3 0}) \mathbf{N}(\%)\end{array}$ & $\begin{array}{l}\text { Number of CNS events }>\mathbf{1 0 0} \text { days } \\
\text { post-transplant }(\boldsymbol{n}=\mathbf{4 7}) \boldsymbol{N}(\%)\end{array}$ \\
\hline PRES & $13(43.3)$ & $4(8.5 \%)$ \\
Leukoencephalopathy & $4(13.3)$ & $6(12.7)$ \\
Venous thrombosis & $3(10)$ & $2(4.2)$ \\
Hemorrhage & $3(10)$ & $5(10.6)$ \\
CNS recurrence & $6(20)$ & $22(46.8)$ \\
Infection & $0(0 \%)$ & $2(4.2)$ \\
Atrophy & $1(3.3 \%)$ & $3(6.3)$ \\
Osmotic demyelination syndrome & $0(0 \%)$ & $1(2.1)$ \\
Mineralizing micro-angiopathy & $0(0 \%)$ & $1(2.1)$ \\
Cortical laminar necrosis & $0(0 \%)$ & $1(2.1)$ \\
\hline
\end{tabular}

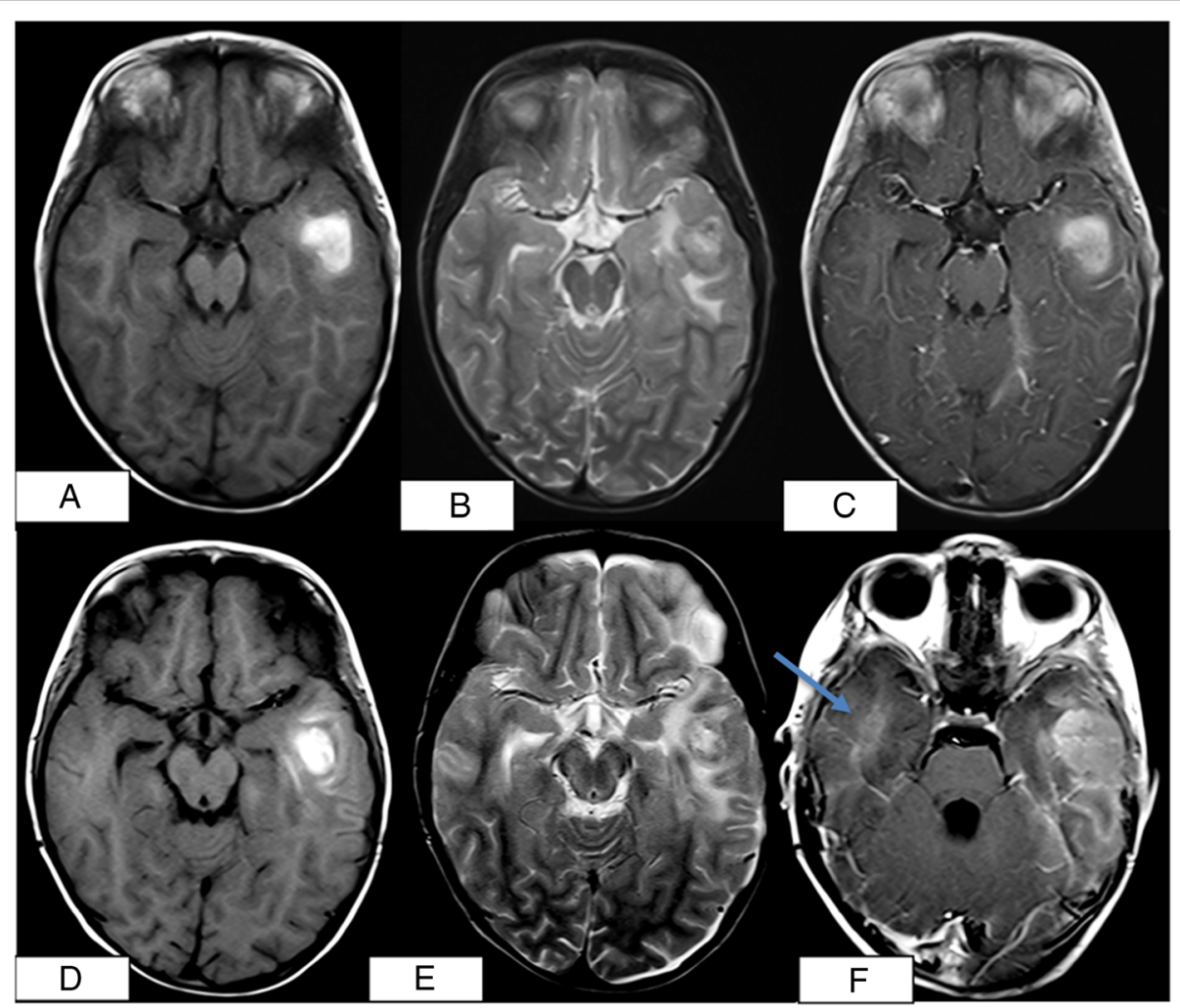

Fig. 2 MRI examinations of a 5-year-old post-allogenic HSCT patient presenting with convulsions. $\mathbf{a}, \mathbf{b}$ and $\mathbf{c}$ Initial MRI examination: axial T1WI (a), axial T2WI (b), and post-contrast axial T1WI (c) sequences revealed left temporal intra-axial SOL showing high T1WI signal, heterogenous T2WI signal, and heterogeneous post-contrast avid enhancement with related mild meningeal enhancement. Subsequent mass effect is noted evident as effacing the overlying sulci, compressing the ipsilateral temporal horn. The patient was initially diagnosed as a case of left temporal intraparenchymal hematoma. Marked deterioration of the patient required follow-up MRI examination after 3 weeks. d, e, and $\mathbf{f}$ Axial T1WI (d), axial T2WI (e), and post-contrast axial T1WI (f) sequences revealed a rather stationary course of the previously noted SOL with markedly evident related meningeal enhancement and newly developed right temporal lobe meningeal enhancement (blue arrow). Pathology after temporal lobectomy revealed relapsed $B$ cell non-Hodgkin lymphoma 


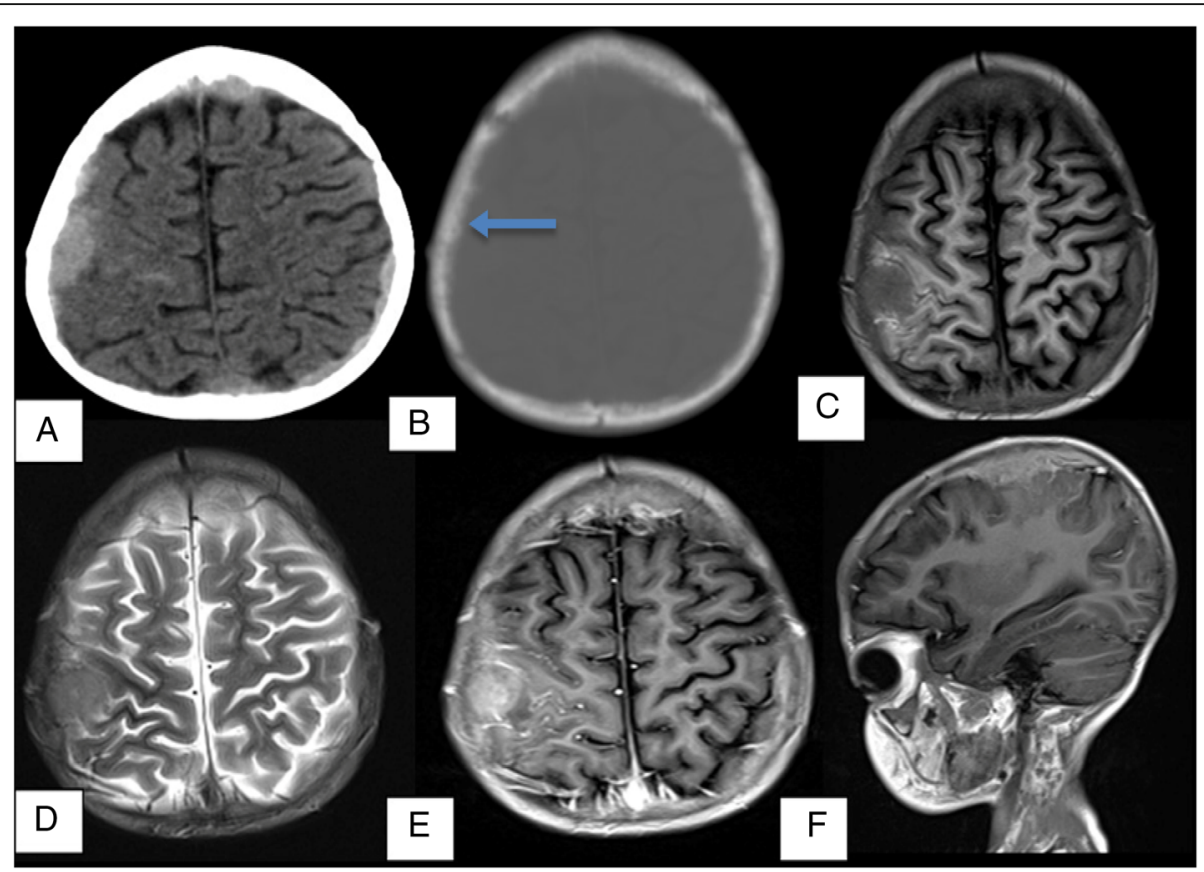

Fig. $3 \mathrm{CT}$ and MRI examinations of a 6-year-old neuroblastoma patient post-autologous HSCT presenting with convulsions. a \& b Axial noncontrast-enhanced CT shows bilateral irregular hyperdense meningeal thickening with underlying osseous erosions (blue arrow). c \& $\mathbf{d}$ Conventional non-enhanced T1 axial (c) and T2 axial (d) images revealing diffuse irregular meningeal thickening mounting to extra-axial SOL formation seen eliciting intermediate signal in both $\mathrm{T} 1$ and $\mathrm{T} 2 \mathrm{Wl}$ sequences with related altered heterogeneous osseous signal. e \& $\mathbf{f}$ Axial (e) and sagittal (f) T1 post-contrast images showing heterogeneous avid enhancement of the meningeal thickening and related extra-axial SOLs. Picture impressive of osseous and meningeal CNS recurrence of neuroblastoma

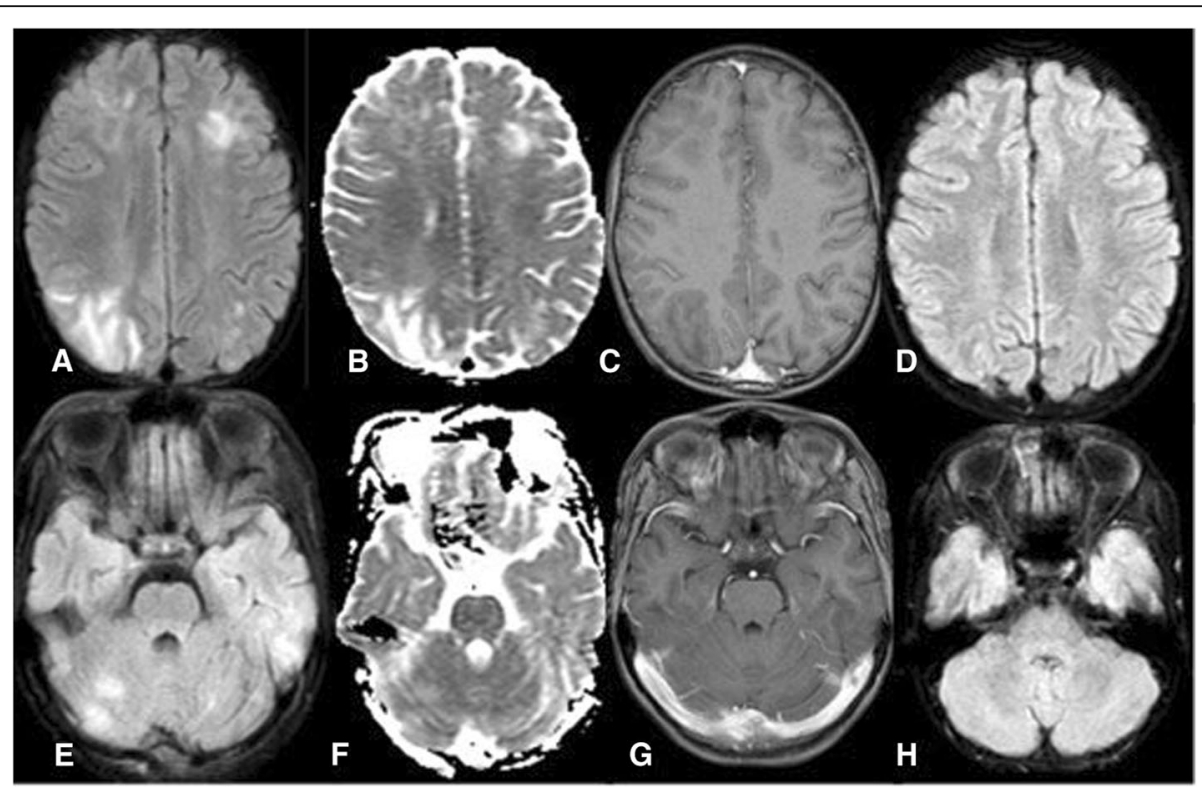

Fig. $4 \mathrm{MRI}$ examination of an 8-year-old post-allogenic HSCT patient presenting with convulsions and disturbed conscious. a \& e axial FLAIR images revealing bilateral frontal, parietal, occipital (not shown in images), and cerebellar asymmetrical cortical and subcortical high FLAIR signal. b \& $\mathbf{f}$ ADC maps showing bilateral frontal, parietal, and occipital high signal impressive of facilitated diffusion. c \& $\mathbf{g}$ Contrast-enhanced axial image showing no evident post-contrast enhancement. The patient was diagnosed with posterior reversible encephalopathy syndrome (PRES). $\mathbf{d}$ $\& \mathbf{h}$ Follow up axial FLAIR images showing total resolution of the previously noted altered cortical and subcortical signal with no residual changes 


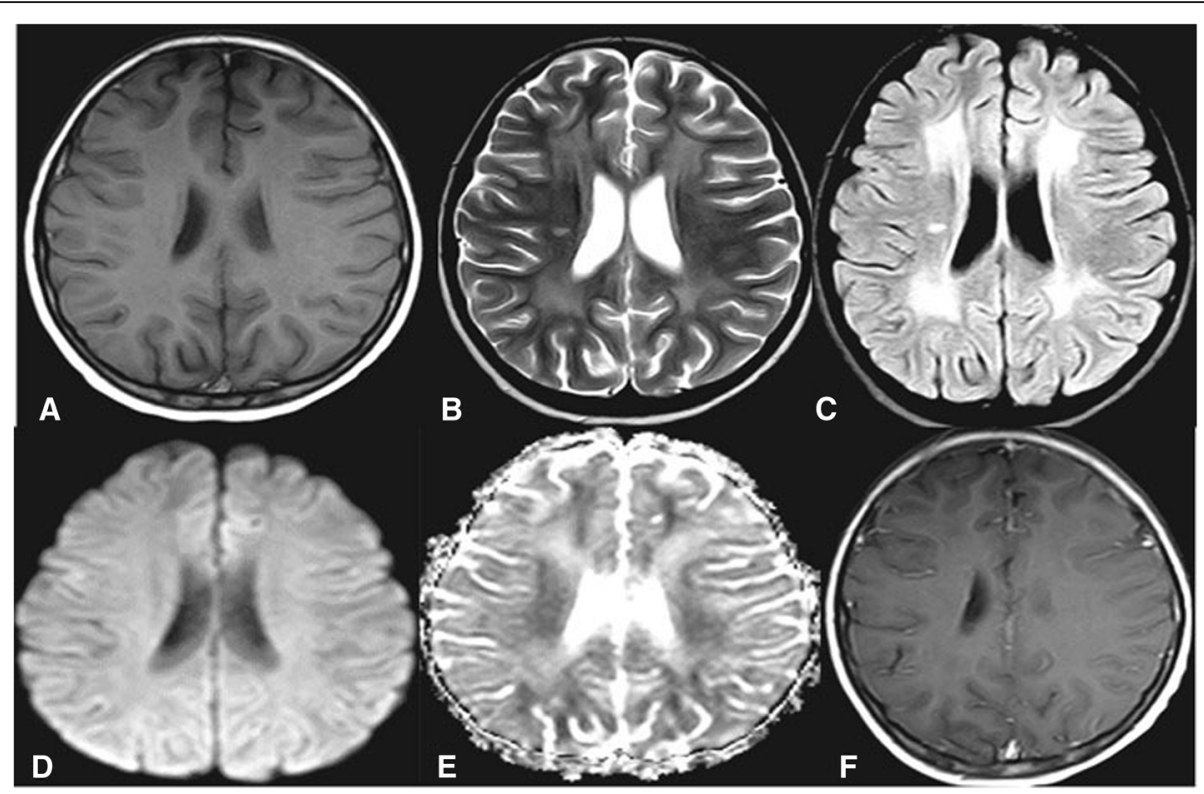

Fig. $5 \mathrm{MRl}$ examination of an 11-year-old post-allogenic HSCT patient presenting with headache. a, b, c Conventional non-enhanced T1 axial (a), T2 axial (b), and FLAIR axial (C) images revealing bilateral rather symmetrical periventricular and centrum semiovale high T2/FLAIR signal appears isointense at T1 images. e, f Bilateral periventricular high signal in DWI $b=1000$ (e) with similar high signal at the ADC map (f). d Contrastenhanced axial image: no evident post-contrast enhancement. Radiological pattern impressive of leukoencephalopathy

intraparenchymal hemorrhage was detected in only $2 / 8$ (25\%) of cases. The hemorrhagic lesions revealed a hyperintense T1WI signal in $80 \%$ of cases and a hyperintense T2WI signal in $60 \%$ of cases.

\section{Leukoencephalopathy (Fig. 5)}

Incidence of post-HSCT leukoencephalopathy is $1.9 \%$, all of which were post-AlloSCT patients with cyclosporine and methotrexate as part of their anti-GVHD regimen. All the cases revealed isodense abnormalities in the $\mathrm{CT}$, requiring further MRI studies. All leukoencephalopathy cases revealed altered hyperintense T2/FLAIR signal with no MRI evidence of diffusion restriction. Leukoencephalopathy shows a stable course in the follow-up examination in $60 \%$ of cases, a progressive course in $20 \%$ of cases, while regression and total resolution of the MRI changes were seen each in $10 \%$ of the cases.

\section{CNS infection}

CNS infection has an incidence of $0.38 \%$, detected in only 2 patients. Both patients were post-AlloSCT, a single case of toxoplasmosis and a single case of limbic encephalitis. The findings were proven using clinicradiological and laboratory correlation.

Only one case of osmotic demyelination syndrome was detected in our study (Fig. 6).

\section{Discussion}

This is a long-term follow-up multifarious study that has included patients with various HSCT types, different initial diagnoses, and different conditioning regimens. Similar studies that discussed CNS complications post-HSCT in both allogenic and autologous HSCT patients are scarce.

Sakellari et al. [8] reported an incidence of postAlloSCT neurological complications ranging from 2.4 to $56 \%$. The reported incidence of neurological complications varies greatly among the different centers due to different factors including the type of transplant, patient population, the definition of neurological complications, duration of follow-up, and study design.

It also varies according to the time the study was conducted, where in the last decades, the increase in the number of patients undergoing transplantation and progression of their overall survival make it more likely to develop unusual complications, such as neurological disorders [8].

In this study, post-AlloSCT CNS complications are more common than those of post-AuSCT; the incidence of post-AlloSCT and post-AuSCT CNSC are 39\% and $11 \%$, respectively. These results were, similar to those detected by Weber et al. [4].

Regarding post-allogenic HSCT, severe acute GVHD is an independent risk factor for the occurrence of CNSC $(\mathrm{OR}=3.3, P$ value $<0.001)$; those results are in concordance with Nam Koh et al. [9] and Sakellari et al. [8] (OR $=11.96 P$ value $<0.001)[10]$. 


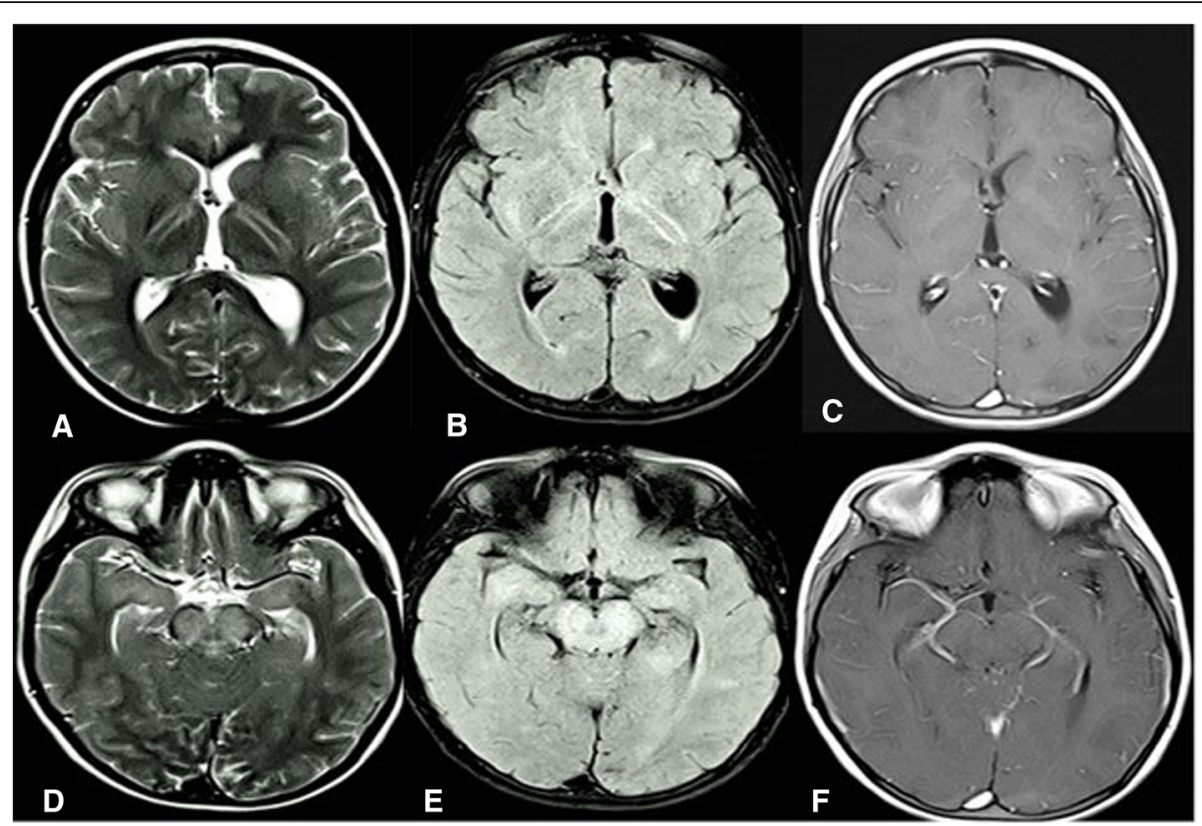

Fig. 6 CT and MRI examinations of a 12-year-old post-autologous HSCT patient presenting with impaired conscious. a, b, d \& e: Axial noncontrast-enhanced T2 images $(\mathbf{a} \& \mathbf{d})$ and FLAIR images $(\mathbf{b} \& \mathbf{e})$ revealing brain stem, bilateral internal and external capsules altered high signal in T2 \& FLAIR images. $\mathbf{c} \& \mathbf{g}$ Axial post-contrast study showing no evidence of post-contrast enhancement at the areas of altered signal. The patient was diagnosed with osmotic demyelination syndrome based on the imaging findings as well as the laboratory finding of low sodium ( $\mathrm{Na}$ ) level

The Chaudhary et al. [10] results regarding the female sex association with a higher likelihood to develop CNSC following AlloSCT were similar to our obtained results.

The most commonly observed CNSC in the current study was CNS disease recurrence (5\%), followed by PRES (3.2\%). However, Sakellari et al. [8] agreed that the most common post-HSCT CNSC is CNS recurrence with a much higher incidence $(17 \%)$ than that detected in our study. Meanwhile, their reported PRES incidence was only $4 \%$.

Recurrent malignancy is the leading cause of death in $14 \%$ of post-HSCT patients [11]. So, it is important to discriminate treatment-induced changes from pathology, post-transplantation lymphoproliferative disorder, and disease recurrence [12].

While this study results match those of Veljanovska et al.'s [13] regarding the incidence of PRES in AlloSCT patients (4.6\%), Bhatt et al.'s [14] revealed a markedly higher incidence (40\%). Cyclosporine was part of the GVHD prevention regimen of all post-AlloSCT patients in the study diagnosed with PRES. No correlation between supra-therapeutic calcineurin inhibitor levels, and PRES is noted.

During the follow-up, PRES was much more common at the $<100$ day-post-HSCT period, during which $70.6 \%$ of PRES episodes occurred. These results are rather comparable to the findings of Veljanovska et al. [13] and
Siegal et al. [15], which revealed that $86 \%$ of PRES episodes occurred during the first 100 days post-transplant.

MRI is still the gold standard in the diagnosis of PRES [16]. Bilateral symmetrical occipital affection was the most common imaging pattern. Atypical PRES sites are comparable to the findings of Chaudhary et al. [10], including the cerebellum, basal ganglia, and thalamus in $29.4,11.4$, and $6 \%$, respectively.

The literature stated that the most common finding for PRES is a high signal on T2WI and FLAIR sequences. This supported the current study results where CT and T1WI MRI findings of our PRES patients were inconclusive, and the main distinctive features were the high T2 and FLAIR signals [13].

Diffusion restriction was detected in $29.4 \%$ of the diagnosed PRES patients, with no definite relation to the signal's reversibility or residual neurological insult. The latest studies revealed that prediction of the outcome of PRES based on DWI (diffusion-weighted imaging) and ADC (apparent diffusion coefficient) values should be used with caution as diffusion restriction is no longer directly linked to PRES irreversibility and permeant neurological insult [16].

Emad-Eldin \& Abdel-Moeti's [17] study of 33 postallogenic HSCT patients with CNS complications revealed a $15 \%$ incidence of leukoencephalopathy; our study including both allogenic and autologous HSCT patients (525) shows a prominently lower incidence of $1.9 \%$; all of them had undergone AlloSCT, and all of 
them had methotrexate in their GVHD prophylaxis protocol.

Literature stated that in patients undergoing HSCT, clinically significant cerebral bleeding was more common than symptomatic thrombotic events where both are important causes of morbidity and mortality [18]. Similarly, our study revealed a higher incidence of post-HSCT hemorrhagic episodes (1.5\%) compared with CVT (cerebral venous thrombosis) episodes (0.9\%).

Compared with our study, Emad-Eldin \& AbdelMoeti [17] and Chaudhary et al. [10], revealed higher incidence of post-HSCT intracranial hemorrhage in 6 and $3.4 \%$, respectively. Moreover, Gerber et al. [19], EmadEldin \& Abdel-Moeti [17], and Hierlmeier et al. [1] reported higher incidences of CVT of $4.6,3$, and $12.2 \%$, respectively.

AlloSCT patients were found to have a higher incidence of CVT and hemorrhagic episodes (1.4 and 2.8\%, respectively) compared to AuSCT patients ( $0.6 \%$ each). Similarly, Hierlmeier et al. [1] and Labrador et al. [20] proved that AuSCT (versus AlloSCT) is protective for HSCT-related thrombocytopenia and CVT.

Maffini et al. [11] stated that subdural hematoma is the most common type of imaging-detected post-HSCT intracranial hemorrhagic episode. Similar results were detected in this study, where subdural hemorrhage was noted in $37.5 \%$ of hemorrhagic episodes.

For imaging assessment of post-HSCT CNS complications in pediatric patients, CT examination is to be avoided whenever possible due to radiation hazards and the fact that its main indication for hemorrhage detection can be superseded by susceptibility imaging. Limited MRI protocols distinctively tailored for each patient are often required to reduce patient under anesthesia time and risk of contrast administration. A proposed imaging protocol is illustrated in Fig. 7.

The limited number of patients in each subgroup of complications restrain proper assessment. Therefore, further studies with larger patient numbers are required for a deeper understanding of the pathogenesis, etiologic factors, risk factors for post-HSCT CNS complications. This may help to design preventive strategies and may refine treatment approaches.

\section{Conclusion}

Hematopoietic stem cell transplant (HSCT) has been increasingly used in the last few decades, with improved success in offering a cure. CNS complications are an important contributor to morbidity and mortality in HSCT patients.

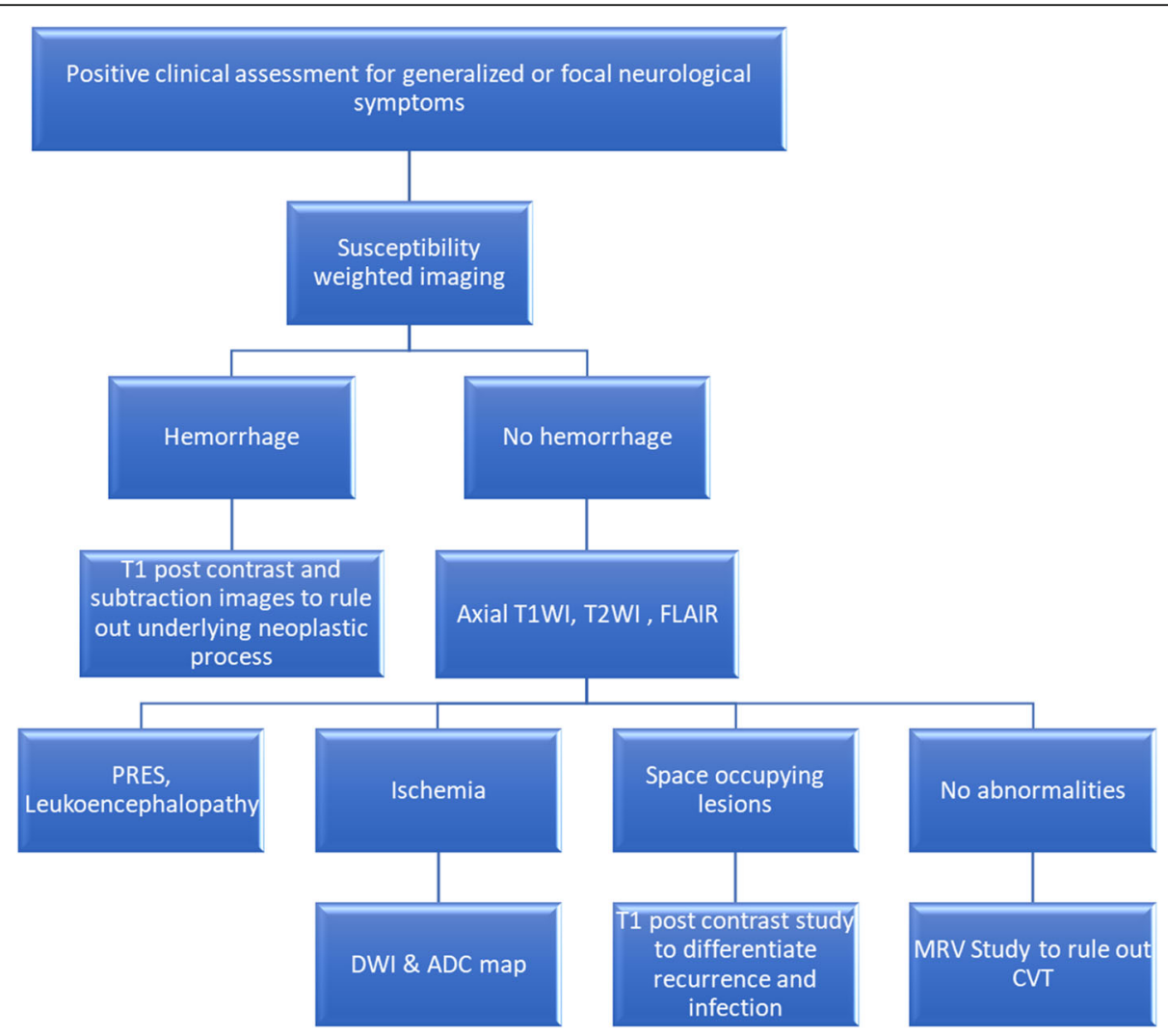

Fig. 7 Proposed imaging protocol for post-HSCT pediatric oncology patients with neurological symptoms 
The incidence of post-HSCT CNSC may be higher in post-allogenic HSCT patients compared with those postautologous HSCT with CNS recurrence, being a more common complication at $>100$ days post-transplant and PRES, being more common at $<100$ days posttransplant. It is essential to maintain a high index of suspicion in this specific subgroup of patients and recognize the specific imaging pattern of each possible complication.

\section{Abbreviations}

AlloSCT: Allogenic stem cell transplant; AuSCT: Autologous stem cell transplant; CNSC: Central nervous system complications; CT: Computed tomography; CVT: Cerebral venous thrombosis; GVHD: Graft versus host disease; HSCT: Hematopoietic stem cell transplant; PRES: Posterior reversible encephalopathy syndrome; MRI: Magnetic resonance imaging

\section{Supplementary Information}

The online version contains supplementary material available at https://doi. org/10.1186/s43055-021-00471-8.

Additional file 1. Learning points.

\section{Acknowledgements}

This research was carried out at Children Cancer Hospital (CCHE-57357) which is fully equipped by modern machines and organized filing system. We want to thanks our colleagues who helped us to do such research work.

\section{Authors' contributions}

$\mathrm{SH}$ wrote the manuscript and is responsible for correspondence to journal. AY collected patient data and was responsible for image processing and collection of patient's images. KA participated in the design of the study and performed the statistical analysis. IZ conceived of the study and participated in its design and coordination and helped to draft the manuscript. $\mathrm{MH}$ and AA were responsible of revision of the draft from clinical point of view. The authors have read and approved the manuscript.

\section{Funding}

This research did not receive any specific grant from funding agencies in the public, commercial, or not-for-profit sectors.

\section{Availability of data and materials}

The datasets used and analyzed during the current study are available from the corresponding author on reasonable request.

\section{Declarations}

\section{Ethics approval and consent to participate}

The study was approved by the Institutional Review Board (IRB) of the National Cancer Institute, Cairo University with ethical committee approval number 201617059.3, session 120 and approval date 26/12/2017. An informed written consent was taken from all subjects.

\section{Consent for publication}

All patients included in this research gave written informed consent to publish the data contained within this study.

\section{Competing interests}

No financial or non-financial competing interests.

\section{Author details}

${ }^{1}$ Department of Diagnostic and Interventional Radiology, National Cancer Institute, Faculty of Medicine, Cairo University and Children's Cancer Hospital (CCHE-57357), Cairo, Egypt. ${ }^{2}$ Department of Pediatric Oncology, National Cancer Institute, Cairo University and Children's Cancer Hospital (CCHE-57357), Cairo, Egypt. 'Department of Clinical Research, Children's Cancer Hospital (CCHE-57357), Cairo, Egypt.
Received: 4 January 2021 Accepted: 23 March 2021

Published online: 08 June 2021

\section{References}

1. HierlmeierS, Eyrich M, Wölfl M, et al (2018) Early and late complications followinghematopoietic stem cell transplantation in pediatric patients - A retrospectiveanalysis over 11 years. PLoS One 13.https://doi.org/10.1371/ journal.pone.0204914

2. DowlingMR, Li S, Dey BR, et al (2018) Neurologic complications after allogeneichematopoietic stem cell transplantation: Risk factors and impact. Bone MarrowTransplant 53:199-206. https://doi.org/10.1038/bmt.2017.239

3. BonardiM, Turpini E, Sanfilippo G, et al (2018) Brain Imaging Findings and NeurologicComplications after Allogenic Hematopoietic Stem Cell Transplantation inChildren. RadioGraphics 38:1223-1238. https://doi.org/1 0.1148/rg.2018170139

4. Weber C, Schaper J, Tibussek D, et al (2008) Diagnostic and therapeuticimplications of neurological complications following paediatric haematopoieticstem cell transplantation. Bone Marrow Transplant 41:253259.https://doi.org/10.1038/sj.bmt.1705905

5. ColomboAA, Marchioni E, Diamanti L, et al (2017) Neurological complications involvingthe central nervous system after allogeneic hematopoietic stem celltransplantation during a period of evolution in transplant modalities: A cohortanalysis. Transplantation 101:616-623.https:// doi.org/10.1097/TP.0000000000001257

6. DulameaAO, Lupescu IG (2018) Neurological complications of hematopoietic celltransplantation in children and adults. Neural Regen. Res. 13:945-954

7. NishiguchiT, Mochizuki K, Shakudo M, et al (2009) CNS complications of hematopoietic stemcell transplantation. Am J Roentgenol 192:1003-1011. https://doi.org/10.2214/AJR.08.1787

8. Sakellaril, Gavriilaki E, Papagiannopoulos S, et al (2019) Neurological adverse eventspost allogeneic hematopoietic cell transplantation: major determinants ofmorbidity and mortality. J Neurol 266:1960-1972.https://doi. org/10.1007/s00415-019-09372-3

9. NamKoh K, Park M, Eun Kim B, et al (2010) Early central nervous systemcomplications after allogeneic hematopoietic stem cell transplantation inchildren. KOREAN J Hematol Korean J Hematol 45:164-70. https://doi.org/10.5045/kjh.2010.45.3.164

10. ChaudharyRK, Dhakal P, Aryal A, Bhatt VR (2017) Central nervous system complicationsafter allogeneic hematopoietic stem cell transplantation. Futur. Oncol.13:2297-2312

11. MaffiniE, Festuccia M, Brunello L, et al (2017) Neurologic Complications afterAllogeneic Hematopoietic Stem Cell Transplantation. Biol. Blood MarrowTransplant. 23:388-397

12. StoneTJ, Misra SP, Mckinstry lii RC, Vo KD Imaging of Central Nervous SystemComplications of Hematopoietic Stem Cell Transplant: A Chronologic Approach toPathology and Implications for Management. https://doi.org/1 0.3174/ng.4150121

13. VeljanovskaAP, Stojanoski Z, Chadievski L, et al (2019) Posterior ReversibleEncephalopathy Syndrome (PRES) in Children Undergoing Allogeneic Stem CellTransplantation. Prilozi 40:81-86. https://doi.org/10.24 78/prilozi-2019-0007

14. BhattVR, Balasetti V, Abduall JJ, et al (2015) Central Nervous System Complicationsand Outcomes After Allogeneic Hematopoietic Stem Cell Transplantation. Clin LymphomaMyeloma Leuk. https://doi.org/10.1016/j. clml.2015.06.004

15. SiegalD, Keller A, Xu W, et al (2007) Central Nervous System Complications afterAllogeneic Hematopoietic Stem Cell Transplantation: Incidence, Manifestations,and Clinical Significance. Biol Blood Marrow Transplant 13: 1369-1379.https://doi.org/10.1016/j.bbmt.2007.07.013

16. WagihA, Mohsen L, Rayan MM, et al (2015) Posterior reversible encephalopathysyndrome (PRES): Restricted diffusion does not necessarily meanirreversibility. Polish J Radiol 80:210-216. https://doi.org/10.12659/PJR. 893460

17. Emad-Eldin S, Abdel-Moeti M (2014) Brain MR imaging abnormalities in pediatric patients after allogeneic bone marrow transplantation. Egypt J Radiol Nucl Med 45:1265-1274. https://doi.org/10.1016/j.ejrnm.2014.07.005

18. Zahid MF, Murad MH, Litzow MR, et al (2016) Venous thromboembolism following hematopoietic stem cell transplantation - a systematic review and meta-analysis. Ann. Hematol. 95:1457-1464 
19. Gerber DE, Segal JB, Yair Levy M, et al (2008) The incidence of and risk factors for venous thromboembolism (VTE) and bleeding among 1514 patients undergoing hematopoietic stem cell transplantation: Implications for VTE prevention. Blood 112:504-510. https://doi.org/10.1182/blood-2 007-10-117051

20. Labrador J, Lopez-Anglada L, Perez-Lopez E, et al (2013) Analysis of incidence, risk factors and clinical outcome of thromboembolic and bleeding events in 431 allogeneic hematopoietic stem cell transplantation recipients. Haematologica 98:437-443. https://doi.org/10.3324/haematol.2 012.069559

\section{Publisher's Note}

Springer Nature remains neutral with regard to jurisdictional claims in published maps and institutional affiliations.

Submit your manuscript to a SpringerOpen ${ }^{\circ}$ journal and benefit from:

- Convenient online submission

- Rigorous peer review

- Open access: articles freely available online

High visibility within the field

- Retaining the copyright to your article

Submit your next manuscript at $\boldsymbol{\nabla}$ springeropen.com 